\title{
Attention and consolidation as factors in retention
}

EDWARD LEVONIAN

The skin resistances of Ss (61 high school students) were recorded during a 10-min. instructional film, and $S s$ were tested for retention of information immediately after (shortterm) and $1 \mathrm{wk}$. after (long-term) the film. Resistance decrements which preceded information presentation (pre-decrements) led to short-term and long-term retention, whereas post-decrements led to reminiscence. These results were interpreted in terms of attention and consolidation.

It has been demonstrated (Sokolov, 1963) that the orienting reflex (OR) is followed by an increase in the analyzer sensitivity of the $\mathrm{S}$. It is assumed here that this increase in perceptual sensitivity will enhance attention, which in turn will facilitate the reception and retention of information which is presented immediately after the occurrence of an OR.

It has also been demonstrated (Kleinsmith \& Kaplan, $1963,1964)$ that arousal which follows the onset of information presentation inhibits short-term retention and facilitates long-term retention. It is assumed here that these results are attributable to a process involving consolidation of the memory trace.

The effects of the $O R$ and arousal on information retention are more readily considered in terms of a common index. One component of the OR is the galvanic skin response (GSR), which is also used frequently as a measure of arousal. In terms of this common measure, the two considerations above lead to the prediction that a GSR which precedes information presentation will facilitate information retention, presumably both short-term and long-term, whereas a GSR which follows information presentation will inhibit short-term retention and facilitate long-term retention.

In this study the term "resistance decrement," or simply "decrement," is used instead of GSR. A "pre-decrement" is one which precedes information presentation; a "post-decrement" follows information presentation. Specifically, the prediction is made that if the $S$ 's pre-decrement is larger than his post-decrement, he will show both short-term and long-term retention of the presented information, whereas if the S's post-decrement is larger than his pre-decrement, he will show long-term but not short-term retention of the presented information.

Method

Ss consisted of students enrolled in a classroom course in driver education in a public high school in Los Angeles. About half the students were female, and essentially all were 15 years old. There were four consecutive classes, and each met in the same room- two classes before lunch, two after lunch. Data were recorded in the room and at the time the classes normally met.

The Ss viewed a 10-min. film on traffic safety which had previously been scheduled by the teacher as an integral part of the course. Immediately thereafter (short-term) the Ss responded to a questionnaire which included 15 items pertaining to auditory or visual information presented at 15 points in the film. The Ss were thanked and given the impression that their involvement in the study was over. A week later (long-term) they were asked to respond once more to the same questionnaire.

Skin resistance was recorded during the film. Each $S$ wore a pair of finger-tip electrodes on the index and ring fingers of his non-writing hand (Levonian, 1963). A constant current of 2 microamps was passed through the skin, and the resulting voltage was sampled, amplified, recorded on magnetic tape, and transferred automatically to computer by a procedure described previously (Levonian, 1962). The film was projected at the standard rate of 24 frames per second, and for each of the $16080 \mathrm{film}$ frames one skin resistance value was recorded from each $\mathrm{S}$. These 16080 values were standardized to zero mean and unit variance for each S. 1

Ss were identified who showed both short-term and long-term retention for at least one item (referred to here as a retention item) as well as long-term but not short-term retention for at least one other item (reminiscence item). There were 61 such Ss, and only their data were involved in this study. Preand post-decrements were measured for each such item. The pre-decrement was taken as the maximum drop (including zero) during the $30 \mathrm{sec}$. preceding the midpoint of information presentation; a post-decrement was defined similarly for the 30 sec. following the midpoint. The interval over which information was presented was never more than 4 sec.

If the $\mathrm{S}$ yielded more than one retention item, his pre-decrement (and post-decrement) was taken as the average of the pre-decrements (and post-decrements) for all retention items; an identical procedure was followed for his reminiscence items.

Results

Results are tested for significance at the .01 level.

The predictions being tested here are (a) that for his retention item, the S's pre-decrement will be larger (more of a drop) than his post-decrement, and (b) that for his reminiscence item, the S's post-decrement 
will be larger than his pre-decrement. Only one in four Ss would fulfill both predictions if retention and reminiscence items were not related to the relative magnitude of pre- and post-decrements. In fact, 25 of the 61 Ss confirmed both predictions. The difference between obtained and chance-expected frequencies results in a chi square of 8.30 , which is significant. Discussion

It seems reasonably clear that the temporal relation of resistance decrement to information presentation is related to information retention such that a pre-decrement is associated with good short-term and good long-term retention, whereas a post-decrement is associated with poor short-term but good long-term retention. However, these results do not prove that the first effect is due in part to attention, the second to consolidation, as is proposed here, for independent measures of attention and consolidation were not obtained in this study. Nevertheless, the temporal relation of resistance decrement to information presentation in the two cases suggests that the first effect is due in part to a perceptual process, the second effect to a post-perceptual process.

The possible nature of these processes is discussed most readily by considering first the process which is reflected by a post-decrement. It is assumed here that the process which initiates the post-decrementalso initiates activity in widespread areas of the cortex through reticular support. This activity results in the contribution of transmitter substances at synapses in the assembly of cells (Hebb, 1949) involved in the memory trace by excitatory neurons which are not in the locus of the memory trace but which terminate at synapses which are. Such summation is assumed to result in a reduction in the amount of transmitter substances which must be supplied by a neuron in the locus of the memory trace to bring its axonal synapse to the transmission threshold. This results in a reduction in the minimally-necessary firing rate of this neuron, for the amount of transmitter substances is determined by the neuron's firing rate. Since the vesicles at the axonal terminal contain only a limited amount of transmitter substances (Eccles, 1965), and since these can be replaced only after considerable delay (Dingman \& Sporn, 1964), it follows that a lower firing rate will be associated with a longer firing duration. Under the assumptions that consolidation rate is proportional to firing rate, and that consoli- dation is proportional to the integral of consolidation rate over time, a large post-decrement should result in poor initial consolidation but enhanced eventual consolidation. The process described in this paragraph is assumed to be the basis for the finding that a large post-decrement is associated with poor short-term but good long-term retention.

Presumably this same process operates with the occurrence of a pre-decrement, in which case longterm retention would be expected. However, the effect of this consolidation process is confounded with the effect of a perceptual process associated with the occurrence of a pre-decrement. This perceptual process increases the sensitivity of the $S$ to signal stimulifilm information in the case of the current study-and, presumably, this directed sensitivity constitutes the physiological basis of attention. This enhanced sensitivity is assumed to be the basis for the finding that a large pre-decrement enhances short-term retention.

In summary, it is suggested that the occurrence of a pre-decrement is associated with attention and consolidation processes leading to, respectively, short-term and long-term retention, whereas the occurrence of a post-decrement is associated only with the consolidation process, leading only to long-term retention.

\section{References}

Dingman, W., \& Spom, M. B. Molecular theories of memory, Science, 1964, 144, 26-29.

Eccles, J.. The synapse. Scient. American, 1965, 212, 56-66. Hebb, D. O. The organization of behavior. New York: Wiley, 1949. Kleinsmith, L. J., \& Kaplan, S. Paired-associate learning as a function of arousal and interpolated activity. J. exp. Psychol, $1963,65,190-193$

Kleinsmith, L. J., \& Kaplan, S. The interaction of arousal and recall interval in nonsense syllable paired-associate learning. J. exp. Psychol., 1964, 67, 124-126.

Levonian, E. Measurement and analysis of physiological response to film. Los Angeles: Univ. of California, Dept. of Engrg. Rep. No. 62-66, 1962.

Levonian, E. Skin resistance electrode for classroom use. Psychol. Rep., 1963, 13, 451-460.

Sokolov, E. N. Perception and the conditioned reflex. New York: Macmillan, 1963.

\section{Note}

1. Data analysis was performed at the UCLA Computing Facility with funds from the California Transportation Agency and the Bureau of Public Roads, U. S. Dept. of Commerce. 